\title{
Article \\ The influenza virus RNA-polymerase and the host RNA-poly- merase II: RPB4 is targeted by a PB2 domain that is involved in viral transcription
}

\author{
Jessica Morel ${ }^{1}$, Laura Sedano ${ }^{1}$, Nathalie Lejal ${ }^{1}$, Bruno Da Costa ${ }^{1}$, Eric Batsché ${ }^{2}$, Christian Muchardt ${ }^{2}$ and Bernard \\ Delmas ${ }^{1, *}$ \\ 1 VIM, INRAE, UVSQ, Université Paris-Saclay, Jouy-en-Josas, France; jessica.morel@inrae.fr (J.M.) ; laura.se-

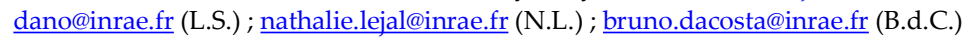 \\ 2 UMR8256 CNRS, Institut Biologie Paris Seine, Sorbonne Université, Paris , France; eric.batsche@sorbonne- \\ universite.fr (E.B.) ; christian.muchardt@sorbonne-universite.fr (C.M.) \\ * Correspondence: bernard.delmas@inrae.fr ;
}

\begin{abstract}
Influenza virus transcription is catalyzed by the viral RNA-polymerase (FluPol) through a cap-snatching activity. The snatching of the cap of cellular mRNA by FluPol is preceded by its binding to the flexible C-terminal domain (CTD) of the RPB1 subunit of RNA-polymerase II (Pol II). To better understand how FluPol brings the 3'-end of the genomic RNAs in close proximity to the host-derived primer, we hypothesized that FluPol may recognize additional Pol II subunits/domains to ensure cap-snatching. Using binary complementation assays between the Pol II and FluPol subunits and their structural domains, we revealed an interaction between the N-third domain of PB2 and RPB4. This interaction was confirmed by a co-immunoprecipitation assay and found to occur with the homologous domains of influenza B and C FluPols. Residues [1-72] of RPB4 were found critical in this interaction. Numerous punctual mutants generated at conserved positions between influenza A, B and C FluPols in the N-third domain of PB2 exhibited strong transcriptional activity defect. These results suggest that FluPol interacts with several domains/subunits of Pol II, the CTD to bind Pol II initiating host transcription and a second on RPB4 to locate FluPol at the proximity of the $5^{\prime}$-end of nascent host mRNA.
\end{abstract}

Keywords: influenza virus; RNA-polymerase; RNA-polymerase II; protein-protein interaction; PPI; cap snatching; transcription; binary complementation assay

\section{Introduction}

Influenza A viruses (IAVs) are important viral respiratory pathogens of humans, members of the Orthomyxoviridae family. These viruses contain a negative-sense singlestranded segmented RNA genome (reviewed in [1]). The three largest segments encode the subunits of the viral RNA-dependent RNA polymerase (FluPol), including the two basic proteins PB1 and PB2 and the acidic subunit PA [2]. Unlike observed for many other RNA viruses, the IAV genome is transcribed and replicated in the nucleus of infected cells, where the FluPol nucleotide polymerization activity carries out both replication and transcription. During the latter, an additional "cap-snatching" function is implemented to steal short 5'-capped RNA primers from host mRNAs [3].

In the viral particle, the eight viral RNA genomic segments are packaged together with numerous copies of the viral nucleoprotein (NP) and of FluPol in viral ribonucleoprotein complexes (vRNPs). After binding to sialic acid on the cell surface, virions are endocytosed, and fused with the endosomal membrane. vRNPs are then released into the cytoplasm and transported to the nucleus where they start synthesizing viral mRNAs to produce viral proteins. Thus, FluPol transcribes each vRNA genome segments, producing 
positive sense viral mRNA with a 5'-terminal N7-methyl guanosine (m7G) cap and a 3'polyA tail. FluPol which does not possess capping activity binds to $\mathrm{m} 7 \mathrm{G}$ on nascent host RNAs using a cap-binding domain on PB2. Then, it cleaves the host mRNA approximately 10 - to 15 nucleotides downstream of the 5'-cap, using the PA endonuclease. The resulting short 5' capped RNA fragment acts as a primer to initiate transcription of the viral genome segments. Polyadenylation is carried out by a FluPol stuttering at a 5'-proximal poly(U) polyadenylation signal present on each genomic vRNA [4]. The complete FluPol transcription activity (initiation, elongation, polyadenylation and FluPol recycling) has been characterized at near atomic resolution $[5,6]$.

Cap snatching by FluPol requires interaction(s) with the host transcriptional machinery [7-9]. The host RNA-polymerase II (Pol II), a complex of 12 subunits (RPB1-12), synthesizes cellular mRNAs (and diverse noncoding RNAs) which are 5'-m7G capped. Its catalytic center is borne by the two largest subunits (RPB1 and RPB2), while smaller subunits (RPB3 to RPB12) are arrayed at the periphery [10,11]. RPB1 in mammals has a large mobile C-terminal domain (CTD) composed of 52 heptad repeats (consensus sequence Tyr-Ser-Pro-Thr-Ser-Pro-Ser) that recruit transcription factors. Residues of the CTD are subjected to post-translational modifications, the most common being the phosphorylation of Ser2 and/or Ser5. The Ser5P CTD is recognized by the nuclear machinery involved in early steps of mRNA transcription, but remains detectable throughout the gene body [12]. FluPol interacts with RPB1 by binding to its CTD, and more specifically to heptad repeats carrying the Ser5P modification [13]. This interaction has been structurally defined with CTD mimic Ser5P peptides and FluPols of influenza A, B and C viruses [14,15]. These studies document a bipartite binding site on the CTD, while the involved FluPol surfaces appear imperfectly conserved between influenza virus subtypes.

In this study, to better define the molecular mechanisms underlying the FluPol capsnatching activity, we explored whether FluPol may interact with Pol II via domains or subunits other than the CTD of RPB1. We thus used binary complementation assays to systematically investigate effective interactions between FluPol and Pol II subunits. This approach allowed identification and validation of an interaction between the third N-ter domain of PB2 and RPB4. This interaction was conserved among FluPol virus types, and mutations in the implicated domain of the viral protein were found to cripple viral transcription. These results suggest that during viral transcription, FluPol associates with the regulatory CTD domain of RPB1, while also interacting with RPB4, at a site where nascent capped RNAs emerge from Pol II. We speculate that this second complementary anchoring position may participate in the docking of FluPol onto nascent RNAs, as a step preliminary to the "cap-snatching".

\section{Materials and Methods}

\subsection{Cells and virus}

HEK-293T cells were maintained in Dulbecco's modified Eagle's Medium (DMEM) supplemented with 10\% fetal calf serum (FCS), $2 \mathrm{mM}$ L-glutamine, $100 \mathrm{IU} / \mathrm{ml}$ penicillin, $100 \mathrm{mg} / \mathrm{ml}$ streptomycin at $37^{\circ} \mathrm{C}$ and 5\% CO2. Wild-type (wt) and PB2 mutant viruses were generated by reverse genetics using the 12-plasmid reverse genetics system kindly provided by G. Brownlee [16]. Site-directed mutagenesis was carried out on the PB2 gene by using the QuikChange site-directed mutagenesis kit (Stratagene, La Jolla, CA). The viruses were prepared as previously described [17]. Briefly, a 1-day coculture of HEK-293T and MDCK cells (seeding of $3 \times 10^{5}$ and $4 \times 10^{5}$ cells, respectively, in P6 plates) was transfected with a plasmid mixture $(0.25 \mu \mathrm{g}$ per plasmid) using FugeneHD (Promega) according to the manufacturer's recommendations. At $48 \mathrm{~h}$ post-transfection, cell supernatants were harvested and used to inoculate MDCK cells for the production of rescued virus stocks. The FluPol genes of the recombinant viruses were sequenced to validate the presence of engineered mutations. 


\subsection{Protein-protein interaction assays}

\subsubsection{Split-luciferase complementation assay}

A first protein-protein interaction assay was based on the complementation of two trans-complementing fragments of Gaussia princeps luciferase (Gluc), Luc1 and Luc2, as described in Cassonnet et al [18]. Interaction-mediated luciferase activity was measured in cultured cells transiently expressing a protein fused to Gluc1 and another one fused to Gluc2. Codon-optimized of human Pol II cDNAs encoding subunits RPB3, RPB4, RPB5, RPB6, RPB7, RPB8, RPB9, RPB10, RPB11 and RPB12 (all with a HA-tag at their N-terminus) were cloned in pCI-LL-Luc1 and pCI-LL-Luc2 vectors [19], thus resulting to the in frame fusion of the HA-tagged Pol II open reading frame subunits with the Luc1/Luc2 moieties. In these constructs, Pol II subunits are separated from the Luc1/Luc2 moieties by a 10-amino acid long linker (sequence GGGGSGGGGS). The plasmid encoding the RPB2 subunit under the control of the CMV promoter (kindly provided by Benoit Coulombe) was used as a template to insert at the RPB2 3'-end the LL-Luc1/Luc2 moieties. The plasmid pFLAG-Pol-II WT encoding human RPB1 (Addgene number 35175, deposited by Benjamin Blencowe) was used to generate two pCI-LL-Luc1/Luc2 derivates encoding 4 repeats ( $4 \times$ YSPTSPS) of the CTD tail fused to Luc1 or Luc2. FluPol PA, PB1 and PB2 subunits cDNA cloned in pCI-LL-Luc1/Luc2 vectors were kindly provided by Nadia Naffakh [19]. Influenza B and C PB2 cDNAs (which were kindly provided by Wendy Barclay and Sylvie van der Werf, respectively), were used to subclone in frame their 5'-domains with Luc1 in pCI-LL-Luc2 with the Gibson Assembly Master Mix Kit (NEB). Twenty-six pCI-LL-Luc1/Luc2 derivates encoding cellular proteins irrelevant in FluPolPol II interaction were kindly provided by Caroline Demeret. Point mutations and deletions in FluPol cDNAs were generated using Q5 Site Directed Mutagenesis Kit (NEB). HEK-293T cells were seeded at a concentration of 105 cells per well in 48 -well plates. Twenty-four hours post seeding, cells were transfected in duplicate/triplicate with the indicated combinations of pCI-derived plasmids by using polyethylenimine (Polyscience Inc., Le Perray-en-Yvelines, France). In each well, 150 ng of each pCI-LL-Luc1/Luc2 derivate are co-transfected with $150 \mathrm{ng}$ of an empty pCI vector. In several assays, the empty pCI vector was replaced by pCI vectors encoding wild-type FluPol subunits. Twenty-four hours post-transfection, cells were lysed using the Renilla luciferase assay buffer (Promega) for 30 minutes at room temperature. Next, the luciferase enzymatic activity recovered by the assembly of the Luc1 and Luc2 subunits was measured using Renilla luciferase assay reagent (Promega) and a Tecan Infinite 200 PRO luminometer (Renilla luminescence counting program; integration time of $10 \mathrm{~s}$ after injection of ๑๑॰) of the reagent). Normalized luminescence ratios (NLRs) were calculated using pGluc1 and pGluc2 plasmids only encoding Gaussia luciferase moieties as described previously [18].

\subsubsection{Bimolecular fluorescence complementation assay}

BiFC constructs were based on the complementation of two trans-complementing fragments of Venus fluorescent protein, Venus1 (amino acids 1 to 155) and Venus2 (amino acids 156 to 239), as described by Shyu et al [20]. Interaction-mediated fluorescence is observed in cultured cells when a protein A fused to Venus1 interacts with a protein B fused to Venus2. Thus, Pol II and FluPol subunits cDNAs were cloned using adequate restriction sites in pCI-LL-Venus1 and -Venus2 vectors, thus resulting to the in frame fusion of the cloned open reading frames followed by a 10-amino acid long linker (sequence GGGGSGGGGS) with the Venus1/2 moieties. Cells were seeded on coverslips in P12-wells and transfected 24 hours later with combinations of pCI-LL-Venus1/2 derivates (1ug/plasmid). Twenty-four hours after transfection, cells were fixed using $4 \%$ paraformaldehyde for 10 minutes and permeabilized for 15 min using 0,1\% Triton X100. Nuclei were stained with DAPI. The excitation peak used to reveal Venus fluorescence was $515 \mathrm{~nm}$ and its emission was revealed at $528 \mathrm{~nm}$. Images were captured with a confocal microscope and processed with the ImageJ software. 


\subsection{Minireplicon and transcription assays.}

\subsubsection{Minireplicon assay}

HEK-293T cells were seeded in P96 wells (5 x 104 cells/well) and transfected one day later with plasmids driving expression of wild-type or mutated forms of the PA, PB1, PB2 and NP viral proteins with the plasmid pPolI-WSN-NA-firefly luciferase as previously described [21]. Plasmid pMAX-GFP (Ximbio) was used as an internal control for transfection efficiency and data normalization. As a negative control, 293T cells were transfected with PEI with the same plasmids minus the PA expression plasmid. Forty-eight hours post-transfection, cells were lysed in $150 \mu \mathrm{l}$ PLB buffer $(30 \mathrm{mM}$ Tris pH7,9, $10 \mathrm{mM} \mathrm{MgCl}$, $1 \%$ Triton X-100, 20\% Glycerol, 1mM DTT). Luminescence activity was measured with Luciferase Assay System (Promega) on a Tecan Infinite M200Pro luminometer according to the manufacturer's instructions. Replicon activity was quantified by a ratio between the luminescence and the fluorescence signals.

\subsubsection{Transcription assay}

To quantify transcription activity of FluPol mutants, the same procedure than described in the minireplicon assay was used, except that the wild-type PA subunit was replaced by the PA C95A mutant that is deficient for replication [22].

\subsection{Co-immunoprecipitation assay}

HEK-293T cells plated in 6-well plates were transiently transfected with $1 \odot \mathrm{g}$ of pciPB2(36-247)-FLAGtag and/or with 1 ๑g pci-HAtag-RPB4 using PEI. Twenty-four hours post-transfection, cells were rinsed and scrapped at $4^{\circ} \mathrm{C}$ into $1 \mathrm{ml}$ of $50 \mathrm{mM}$ Tris-HCl pH7.4, 2mM EDTA, $150 \mathrm{mM} \mathrm{NaCl}, 1 \%$ Triton X-100, 10\% glycerol supplemented with protease inhibitor (Roche). After $1 \mathrm{~h}$, this material was centrifugated $\left(20 \mathrm{~min}, 12000 \mathrm{~g}\right.$ at $\left.4{ }^{\circ} \mathrm{C}\right)$. Supernatants were collected and incubated under gentle agitation overnight at $4^{\circ} \mathrm{C}$ with 80 @l of a 1:1 slurry of sepharose-IgG beads (GE Healthcare) previously coated with antiFLAG M5 antibody (Sigma-Aldrich). The beads were washed three times with $1 \mathrm{ml}$ of lysis Buffer and once with PBS, then treated for $2 \mathrm{~min}$ at $100^{\circ} \mathrm{C}$ in Laemmli's denaturating buffer plus 5\% 2-mercaptoethanol, and centrifugated. Resulting supernatants were subjected to SDS-PAGE followed by a Western Blot (WB) analysis. An anti-HA antibody conjugated with peroxydase (Roche) and an anti-FLAG antibody conjugated with peroxydase (Sigma-Aldrich) were used to reveal immunoprecipitated products in the WB. The Materials and Methods should be described with sufficient details to allow others to replicate and build on the published results. Please note that the publication of your manuscript implicates that you must make all materials, data, computer code, and protocols associated with the publication available to readers. Please disclose at the submission stage any restrictions on the availability of materials or information. New methods and protocols should be described in detail while well-established methods can be briefly described and appropriately cited.

\section{Results}

\subsection{Novel interactions between FluPol and Pol II subunits}

To identify novel sites of interactions between FluPol and Pol II subunits, we used the previously described Gaussia princeps luciferase-based complementation assay $[18,23]$. In this assay, an interaction between two proteins each fused to either the Luc1 or the Luc2 segments of the Gaussia luciferase enzyme, results in reconstitution of a functional luciferase activity, which can be quantified by addition of substrate (Fig.1A). To favor proper folding of the FluPol and Pol II subunits, the luciferase segments were fused onto their $\mathrm{C}$ terminus. For each sample, the specificity of the interaction over background was estimated by calculating a normalized luminescent ratio (NLR - Fig.1B).

To validate the assay, we first fused each Pol II subunit (from RPB2 to RPB12) with either Luc1 or Luc2, then co-expressed the Luc1-fusions with each of the Luc2-fusions 
challenged (Fig.1C). This approach identified strong and reciprocal contacts between RPB2 and RPB3, RPB2 and RPB12, RPB3 and RPB10, and RPB4 and RPB7. Interaction signal was also identified between RPB12 and RPB3 or RPB10, however in a non-reciprocal manner. Finally, RPB8, RPB10 and RPB12 were found to interact with themselves. Confrontation of these observations with the 3D-structure of Pol II [24] illustrated that the luciferase complementation assay was very efficient at detecting direct interactions between Pol II subunits, while it was oblivious to interactions mediated by the Pol II complex, possibly because of the shortness of the GGGSGGGS linker (summarized by the color code in Fig. 1D).

We next used a similar approach to monitor interactions between FluPol subunits (Fig. 1E). In thse experiments, co-expressed Luc1/Luc2-tagged forms of PA and PB1 yielded NLR signal both in the presence, and in the absence of untagged PB2. In contrast, interaction of PB2 with PA or PB1 was detected only when the three FluPol subunits were co-expressed. As for Pol II, these interactions between FluPol subunits were in agreement with the known 3D-structures of the viral enzyme, and also matched earlier observations on the sequential assembly of in the cytoplasm and in the nucleus $[11,25,26]$.

A.

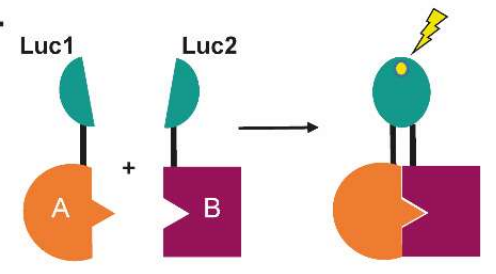

B.

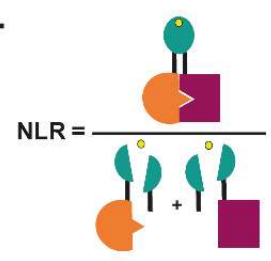

c.
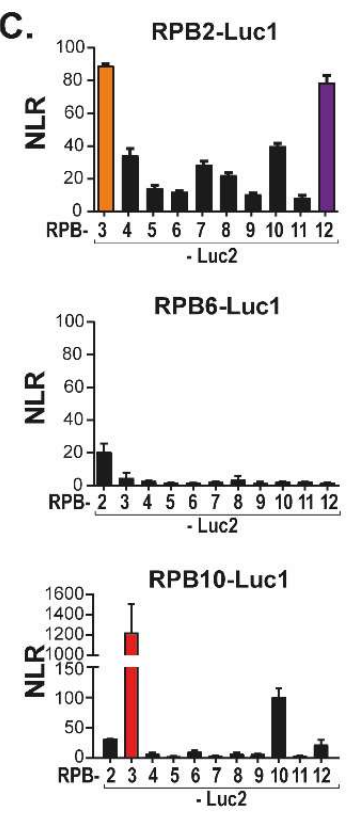
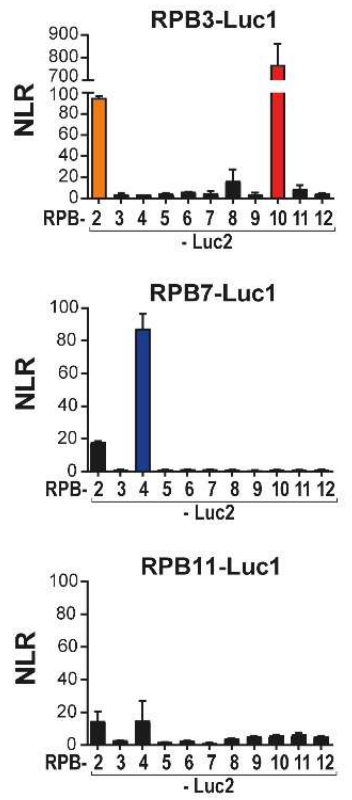
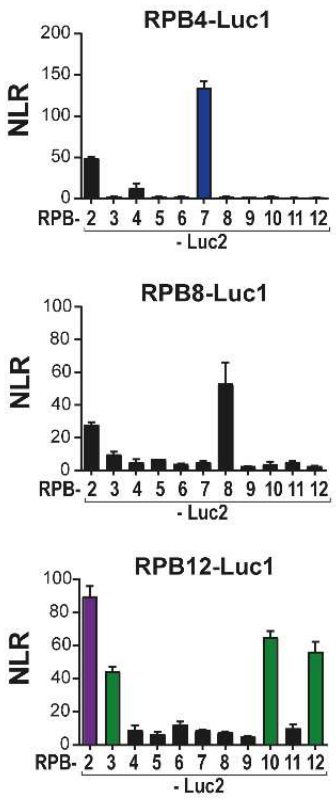
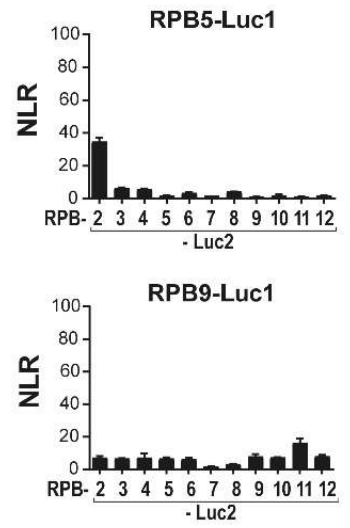

D.

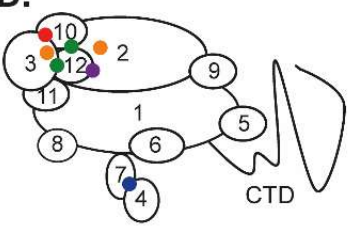

E.

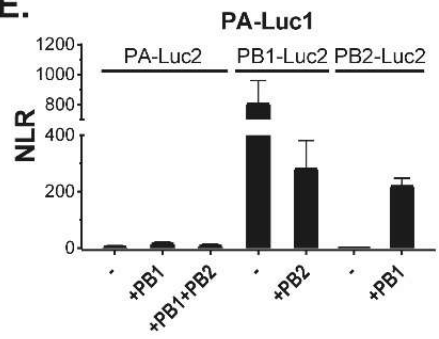

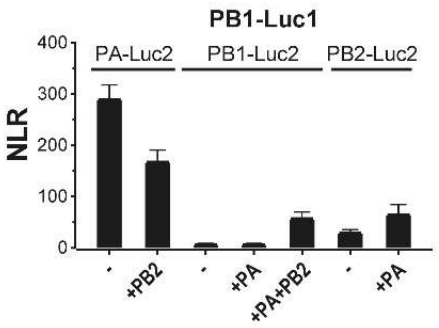

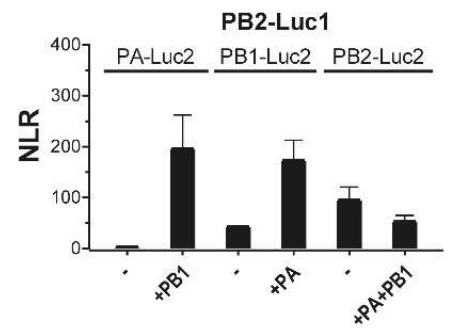


Figure 1. The split-luciferase interaction assay and its validation with Pol II and FluPol subunits. (A) Schematic of the principle of a split-luciferase assay. If protein A end protein B interact, the activity of luciferase is restored and luminescence will be emitted in presence of its substrate. (B) The normalized luminescence ratio (NLR) is calculated as described. (C) NLR values obtained for pairs of Luc1/Luc2-tagged Pol II subunits co-expressed into HEK 293T cells. Twenty-four hours after transfection of plasmids driving Pol II subunits expression, cells were lysed and luminescence was measured. Identical colors indicate reciprocated detected interactions. (D) Pairs of reciprocal interactions are represented by a colored dot on a scheme of Pol II and its subunits. (E) NLR values obtained for pairs of Luc1/Luc2-tagged FluPol subunits plasmids transfected into HEK293T cells with/without untagged FluPol subunits.

Finally, we investigated interactions between FluPol subunits (PA, PB1 or PB2) and Pol II subunits including RPB2 to RPB12, and a fragment of the RPB1 CTD. As expected from structural analyses [14], the RPB1 CTD was found to interact with PA alone and with the assembled FluPol (Fig. Suppl 1, Fig. 2A). With other Pol II subunits, the range of NLR values was 1- to 2-logs of magnitude lower than those observed for internal Pol II and FluPol interactions (Fig. 2B). For each tagged FluPol subunits, RPB3 and RPB4 (and to a less extend RPB7 and RPB8) yielded highest NLR signals when in the presence of an intact FluPol complex. These data were indicative of proximity interactions between FluPol and Pol II not accounted for by RPB1 CTD, an possibly involving RPB3 and RPB4. We noted also that, for the PA bait, the FluPol-Pol II interaction-signals benificited from co-expressed of untagged PB1, but not from coexpression of untagged PB2. Likewise, NLR signals obtained with tagged PB1 and Pol II subunits remained unaffected upon co-expression of untagged PA and PB2. Finally, interactions of the PB2 bait with Pol II subunits were significantly affected by the presence of other FluPol subunits only for RPB4 and RPB7. These data showed that interactions between FluPol and Pol II subunits were not strictly dependent on the assembly of FluPol into an intact complex, and suggested that the assay is amenable to analyze individually the implic\&ation of structurally-defined protein domains of FluPol. 
A.

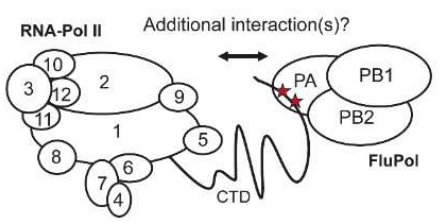

B.
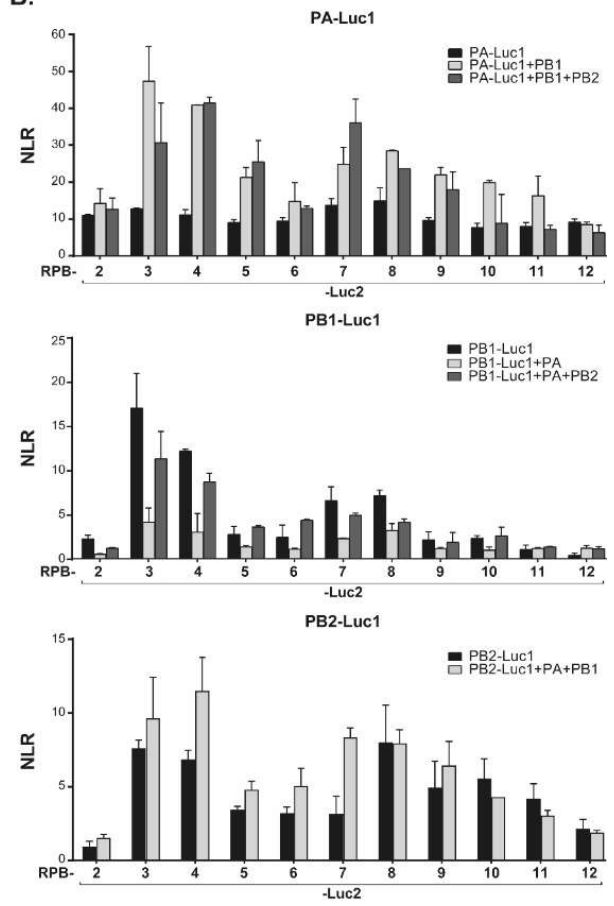

Figure 2. Split-luciferase assays between FluPol and Pol II subunits. (A) Schematic of FluPol and Pol II with their subunits and the interaction between the CTD of Pol II with FluPol. Stars indicated the YSPTSpPS CTD motif (with the phosphorylated Serine 5). (B) Pairs of plasmids encoding a Luc1FluPol subunit with a Luc2-Pol II subunit were transfected into HEK 293T along with/without untagged FluPol subunits. NLR signals were quantified as described in Fig. 1B.

\subsection{The N-terminal region of $P B 2$ mediates interaction with RPB4}

FluPol can be subdivided in functional and structural protein domains. It associates an invariant core comprising the PB1 subunit, stabilized by the PA-linker and the PA-C [amino acid 197 to the C-terminus] domain from the PA subunit, and the PB2-N [amino acid 1 to 247] from the PB2 subunit. This core is then associated with flexibly-linked peripheral domains including the PA endonuclease domain and the two-third C-terminal domain of PB2] [27]. To map the domains of FluPol principally involved in interactions with Pol II, we generated constructs driving expression of corresponding sub-fragments of PA and PB2. In complementation assays with the Pol II subunits, the PA-derived constructs co-expressed with PB1 did not revealed any clear association with Pol II subunits, all combinations yielding very low NLR signals (Fig. 3A). In contrast, co-expression of the core-associated PA domains with a full set of Pol II subunits revealed a strong interaction (NLR score at 85) between PB2-N (residues 1 to 247) and RPB4, not observed with other Pol II subunits (Fig. 3B). The PB2-C (residues 247 to 759) construct also yielded significant NLR scores (approximately 30), but with RPB5 and RPB11. 
A.
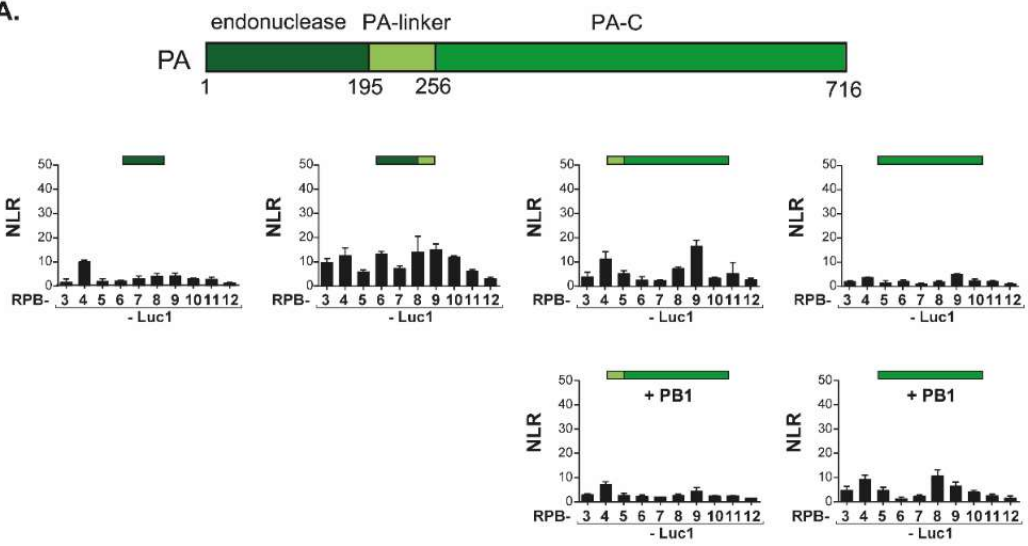

B.

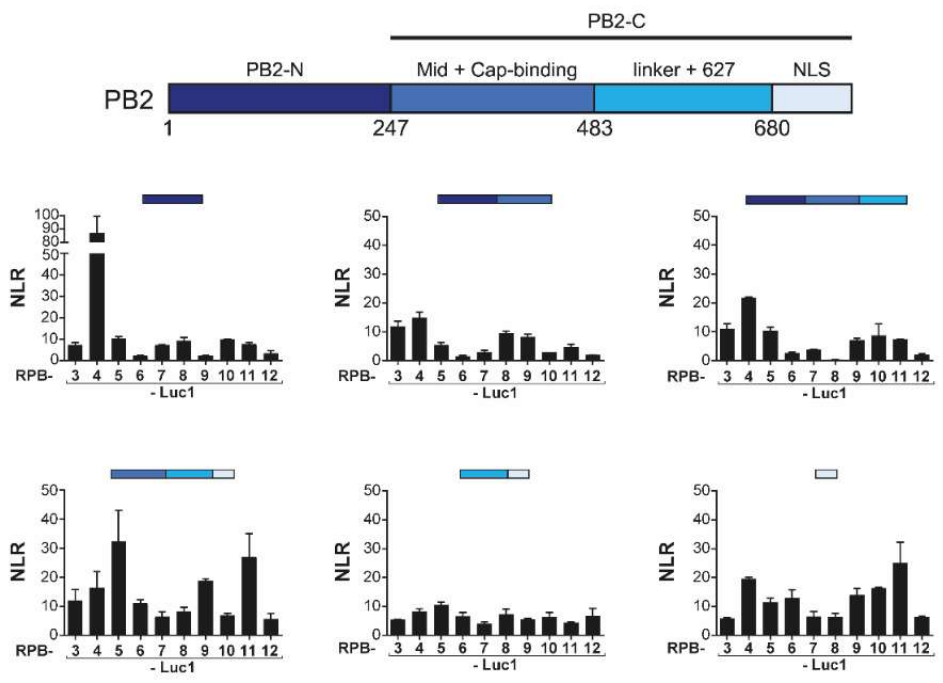

Figure 3. Split-luciferase assays between PA and PB2 domains of FluPol and Pol II subunits. (A) Luc1-PA domains and Luc2-subunits of Pol II were co-expressed into HEK293T cells along with PB1 or an empty plasmid. Twenty-four hours post-transfection, cells were lysed and luminescence was measured. NLR were determined as shown in Fig. 1B. (B) Luc1-PB2 domains and Luc2-subunits of Pol II were co-expressed into HEK293T cells with NLR determined as shown in Fig. 1B.

To validate the specificity of the PB2-N-RPB4 interaction, we next challenged the PB2-N construct against a set of proteins unrelated to the Pol II. In these experiments, neither PB2-N, nor a shorter PB2(36-247) construct lacking 35 N-ter residues interacting with PB1 [28], displayed any interaction with the mock targets (Fig. 4A). Together, these results are indicative of a specific contact between host Pol II subunit RPB4 and the PB2$\mathrm{N}$ domain, while they also suggested auxiliary contacts between RPB5 and RPB11 and the C-terminal regions of PB2.

\subsection{The PB2 RPB4 interaction domain is essential for the virus life cycle}

We next wished to probe the robustness and the biological relevance of the PB2-RPB4 interaction. Co-immunoprecipitation-western assays with epitope-tagged forms of RPB4 and of the PB2(36-247) construct allowed further narrowing down the region of PB2 interacting with RPB4, while also demonstrating that the interaction resisted the relatively stringent conditions characterizing these assays ( $150 \mathrm{mM} \mathrm{NaCl}, 1 \%$ Triton X-100 - Fig. 4B). To further test the interaction under in vivo conditions and with alternative constructs, we generated map the interaction within the cell, we generated a second binary 
complementation assay with PB2 and RPB4 respectively tagged with the N- and C-moieties of the Venus fluorescent protein (Fig. 4C). Co-transfection of these constructs into HEK293T cells resulted in a strong nuclear fluorescence signal, establishing that PB2 can interact with RPB4 under these conditions, while also illustrating that this interaction occurs in the appropriate cellular compartment.

To investigate whether the PB2-RPB4 interaction was confirmed among different influenza virus types, we also constructed a luciferase complementation assay with the PB2 of influenza B and C. In this assay, both PB2(36-247) constructs were found to interact with RPB4, but not with any other Pol II subunit, nor with any of the unrelated target proteins described above (Fig. 4D and 4E).
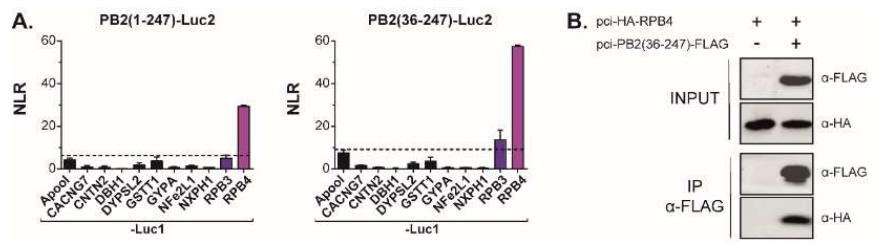

c.

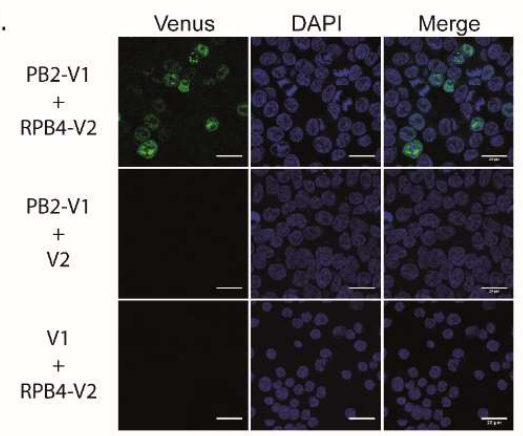

D.
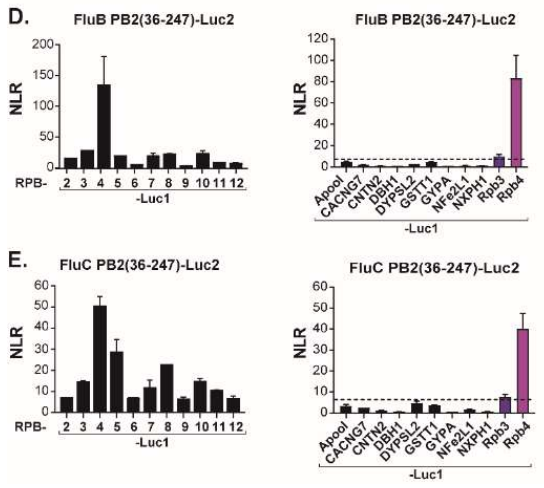

Figure 4. PB2-N domain interacts with the Pol II subunit RPB4. (A) Split-luciferase assays between PB2-N forms (Luc2-PB2(1-247) and Luc2-PB2(36-247) and Luc1-tagged irrelevant proteins. NLR values with these proteins were all found above 9.5. (B) Coimmunoprecipitation assay. Plasmids encoding HA-RPB4 and PB2(36-247)-FLAG were transfected in HEK 293T cells. Twenty-four hours post-transfection, cells were lysed, PB2(36-247)-FLAG was immunoprecipitated and RPB4 was revealed by Western Blotting. (C) Split-Venus complementation assay between PB2 and RPB4. HEK 293T cells were co-transfected with expression plasmids encoding PB2-Venus1, RPB4-Venus 2 and/or with plasmids encoding Venus1 and Venus2 moieties. Cells were fixed 24h later, nuclei were marked with DAPI and fluorescence emitted by reconstituted Venus was measured by confocal microscopy. Bars $=20 \mu \mathrm{m}$. (D and E) The interaction between PB2(36-247) and RPB4 is conserved in influenza types B and C. Plasmids encoding luc2-tagged forms of PB2(36-247) from influenza B (D) or influenza C (E) viruses were co-expressed with Luc1-tagged Pol II subunits were transfected in HEK 293T cells. Twenty-four hours post transfection, cells were lysed and luminescence was measured. NLR values were calculated as described in Fig. 1. 
To further map regions involved in the PB2-RPB4 interaction, we next expressed a series of truncations of PB2-N. When tested in the split-luciferase system, several discrete regions of the PB2 amino acids, stretching from amino acid 130 to 247 were found to yield a signal with RPB4 (Fig. 5). Reciprocally, truncations of RPB4 identified the C-ter moiety of this protein (amino acids 1-72) as essential for the interaction (Fig. 5C). Interestingly, when mapped on the 3D-structures of FluPol and RPB4 [26,29], the protein regions identified in these two sets of deletion mutants matched a series of helixes located at the surface on the two proteins, at a position highly compatible with an implication in proteinprotein interactions.

A.

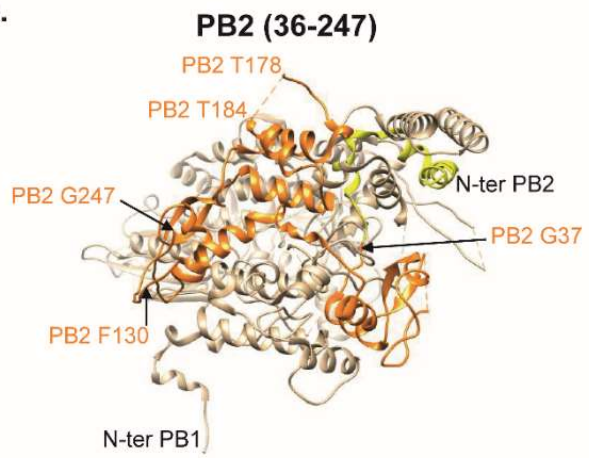

B.

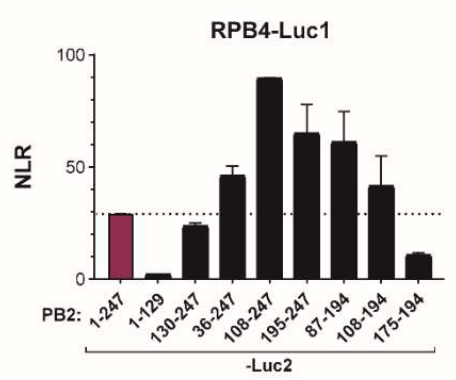

RPB4

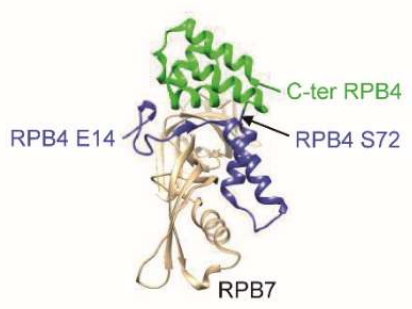

C.

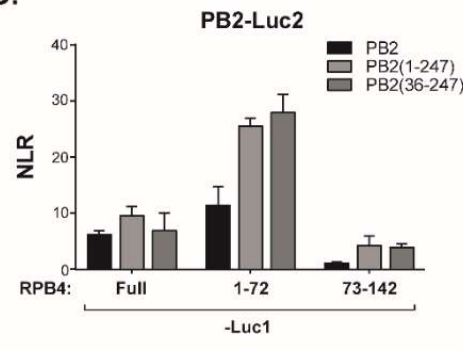

Figure 5. Identification of the PB2 and RPB4 amino acids stretches involved in the FluPol-RPB4 interaction. (A) Structures of the 36-247 domain of PB2 with the PB1 adjacent domain (PDB ID: 6QPF) and of the RPB4/RPB7 dimer (PDB ID: 2C35). (B and C) Split-luciferase complementation assay. (B) Luc2-tagged stretches of PB2(1-247) and Luc1-RPB4 were co-transfected into HEK293T cells. 24h after transfection, cells were lysed and luminescence was measured. (C) The Luc1-tagged N- or Cmoieties of RPB4 were co-expressed with the Luc2-tagged PB2 subunit or with N-ter derived forms of PB2 into HEK293T cells. After cell lysis, luminescence was measured and NLR calculated as in Fig. 1.

Finally, to address the biological function of the PB2 (130-247) region, we engineered a series of fourteen point mutants on residues conserved among influenza $A, B$, and $C$ viruses (Fig. 6A). The activity of these mutants was then assessed in two assays, respectively measuring either the resultant of both replication and transcription $(\mathrm{R}+\mathrm{T})$, or only transcription (T) (Fig. 6B and C). In the latter assay, wild-type PA was replaced by a C95A mutant, rendering FluPol defective for replication [22]. Both assays relied on a pPol1WSN-NA-firefly luciferase plasmid producing a viral RNA (vRNA) in which the coding sequence of the NA gene was replaced by the firefly luciferase gene. This plasmid was transfected into HEK-293T cells together with expression vectors for the appropriate PA construct, the wild-type PB1 and NP constructs, and the various PB2 mutants. The luciferase enzymatic activity measured in extracts of transfected cells and reflecting either $\mathrm{R}+\mathrm{T}$ or T activities dependent on the used PA construct (WT or C95A mutant), revealed that most of the PB2 mutations, with the exception of R144A and E188A, strongly decreased 
FluPol activity in both assays. These observations indicate that this region of PB2 and its conserved residues are critical for viral transcription (Fig. 6C). Finally, to definitively establish the importance of the PB2-N region in the virus cycle, we selected residues 115, 142 and 144 for alanine substitution. Using plasmid-driven reverse genetics, we successfully recovered virus mutants for each of these substitutions. While the R144A virus was found to replicate at high titers $(1.2 \times 107 \mathrm{pfu} / \mathrm{ml}$ in cell culture medium after transfection), the Y115A and the R142A mutants replicated poorly $(6 \times 104 \mathrm{pfu} / \mathrm{ml})$, in accordance with the results obtained in the replication/transcription assays.
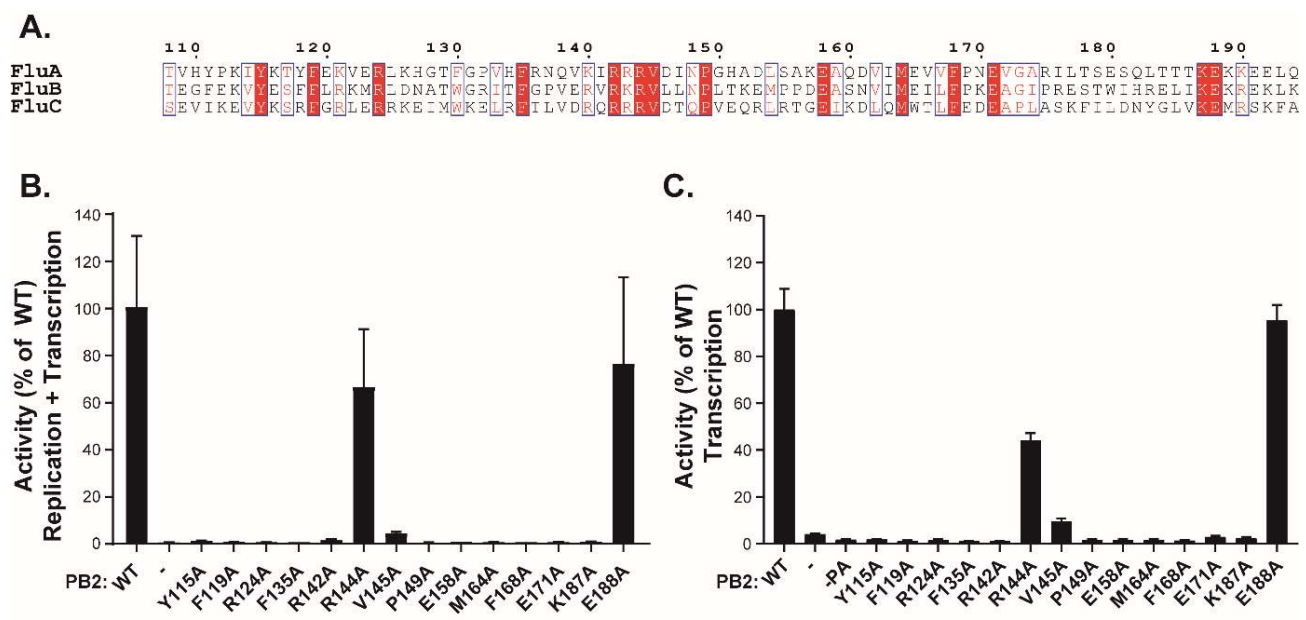

Figure 6. Transcription/replication activity of FluPol with PB2 mutants. (A) Multi-alignment with the PB2 domains (residues 108 to 194) of influenza A, B and C viruses made with ESPript (Robert and Gouet, 2014). Strictly conserved residues are white on a red background, and partially conserved residues are red. (B) Plasmids expressing NP, PB1, PA and wild type or PB2 mutants were cotransfected in 293T cells together with the reporter plasmid WSN-NA-firefly luciferase, allowing the quantification of the polymerase activity. In panel (C), the same procedure than in (B) was used, except that the PA C95A mutant deficient in replicase activity (Serna Martin et al., 2018) was used instead of the wt PA subunit.

\section{Discussion}

The interaction between FluPol and the Pol II complex through the CTD of its RPB1 subunit has been functionally and structurally well-established [9]. This interplay is recognized to allow cap-snatching on nascent mRNA to prime viral mRNA synthesis. However, it remained to elucidate if this binding is sufficient to dock FluPol at the nascent capped RNA or if additional protein-protein interactions on Pol II or with other factors are involved in this process. In this study, we screened potential interactions between FluPol and Pol II subunits. We first found that FluPol can interact with RPB4, one of the eleven other Pol II subunits which is structurally connected to the main body of Pol II through its association with RPB7. Using two binary complementation assays, we evidenced that the N-terminal third of PB2 is involved in the interaction of FluPol with RPB4. We confirmed the existence of this interaction using a co-immunoprecipitation assay. Furthermore, this ability to bind RPB4 was found to be conserved among influenza B and C subtypes, suggesting a critical role of this interaction.

The RPB4/RPB7 heterodimer forms a stalk-like protrusion from the Pol II core complex below a mobile clamp domain, adjacent to the CTD of RPB1 and the RNA exit channel, a position with potential for interactions with multiple components, such as CTDbound factors and modifying enzymes and emerging nascent RNAs [30-36]. In fact, the RPB4/RPB7 stalk has been proposed to act as an additional landing platform for transcription-related factors, with some of which known to also interact with the CTD [37,38]. Thus, 
it is tempting to speculate that the binding of FluPol to RPB4 acts synergistically with its interaction with the CTD to target nascent capped RNAs.

Despite low sequence similarity and identity between the N-terminal third of PB2 among influenza A, B and C viruses, these homologous domains were found to interact with the RPB4 subunit. Fourteen conserved residues in this domain were chosen to evaluate the impact of their substitutions to FluPol transcription activity. Twelve of the fourteen substitutions deeply impacted the transcription activity of FluPol, confirming functionally that the N-ter PB2 domain is important for an efficient interaction with the cell transcription machinery. Therefore, we propose a model (Fig. 7) in which FluPol first targets Pol II via its PA-CTD interaction and then binds to the RPB4 subunit via PB2 to edge closer to the exit canal of Pol II. Thus, the cap-binding domain of PB2 would be in close proximity with nascent mRNAs, hence allowing the cap-snatching by FluPol.

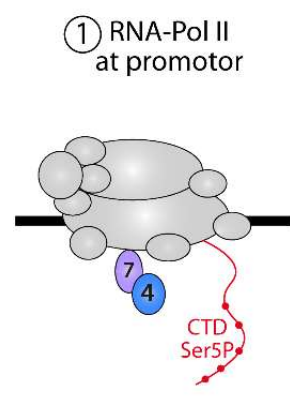

(2) PA targets chromatin
by binding to CTD

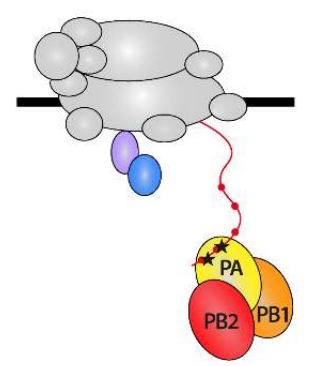

(3) PB2 Nter binds to RPB4 for close proximity with nascent mRNA

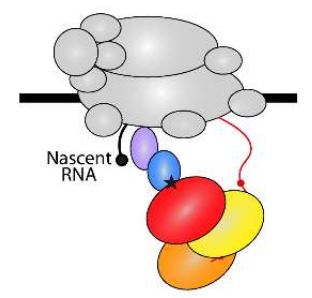

(4) mRNA binds to PB2 cap-binding domain

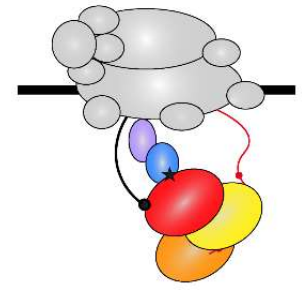

Figure 7. Proposed model for the interaction interplay between FluPol and Pol II. Pol II is represented in gray with RPB4 and RPB7 subunits in blue and lavender, respectively. FluPol first recognizes the YSPTSpPS motifs (with the serine at position 5 phosphorylated) in the CTD of RPB1 to bind the RPB4 subunit located at the vicinity of the Pol II pore that extrudes nascent mRNA during transcription. PB2 binds the cap of the mRNA by its cap-binding domain.

What could be the timing of the FluPol - RPB4/RPB7 dimer interaction? Nascent transcript capping occurs immediately after the emergence of the 5'-end triphosphate from the RNA exit tunnel and is concomitant with promoter-proximal pausing when RNAPol II is bound to NELF and DSIF [39,40]. Shortly after cap completion, the interaction of the cap-binding complex (CBC) to a NELF subunit is consistent with its targeting to the RNA Pol II paused complex, and increases by 100-200-fold the affinity for the modified $5^{\prime}$ end of the transcript. Since the CBC has a higher affinity to the cap structure than FluPol, we propose that FluPol binding to Pol II through interactions its RPB1 CTD and RPB4/RPB7 blocks CBC recruitment or its access to nascent 5'-capped Pol II transcripts. It is also possible that FluPol inhibits the binding of NELF to Pol II or its association to CBC. We are currently investigating these hypotheses to better understand how FluPol controls efficiently capping of viral mRNAs. Authors should discuss the results and how they can be interpreted from the perspective of previous studies and of the working hypotheses. The findings and their implications should be discussed in the broadest context possible. Future research directions may also be highlighted.

Supplementary Materials: The following supporting information can be downloaded at: www.mdpi.com/xxx/s1, Figure S1: title; Table S1: title; Video S1: title. 


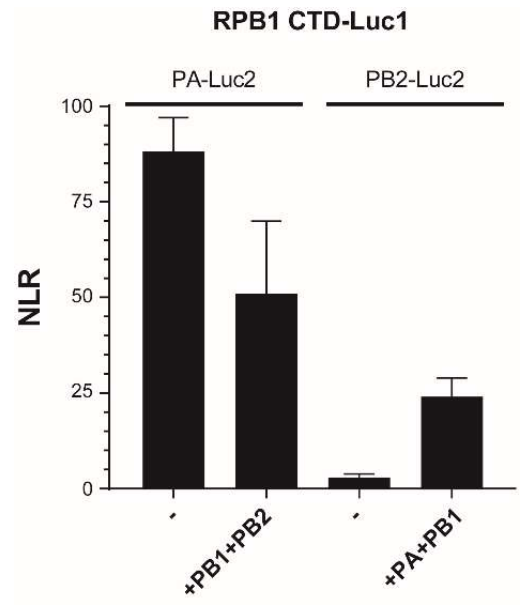

Figure S1. PA interacts with the CTD domain of RPB1. HEK 293T cells were transfected with plasmids encoding the CTD domain of RPB1 fused the Luc1 moiety and plasmids encoding PA or PB2 fused to the Luc2 moiety. Plasmids encoding untagged PA, PB1 or PB2 were also added when indicated. Twenty-four hours post-transfection, cells were lysed and luminescence was measured to calculate NLR values. Data are shown as mean +/- SD.

Author Contributions: Conceptualization, B.D. and C.M.; methodology, B.D. and J.M.; validation, J.M., N.L. and B.D.; formal analysis, J.M. and B.D.; investigation, J.M., L.S., N.L., B.d.C., E.B.; resources, C..M. and B.D.; data curation, J.M. and B.D.; writing-original draft preparation, J.M. and B.D..; writing - review and editing, J.M., C.M. and B.D..; visualization, J.M.; supervision, B.D.; project administration, B.D.; funding acquisition, C.M. and B.D. All authors have read and agreed to the published version of the manuscript."

Funding: This research was funded by the French Agence Nationale de la Recherche (ANR), grant number ANR-17-CE18-0006-01 and the INRAE Animal Health Division.

Data Availability Statement: Data supporting reported results can be provided by B.D.

Acknowledgments: We thank G. Brownlee for the gift of the reverse genetics system of the IAV WSN strain, Benoit Coulombe for the gift of a plasmid encoding human RPB2, Benjamin Blencowe for the plasmid encoding human RPB1 (Addgene number 35175), Nadia Naffakh for the gift of plasmids pCI-Luc1/Luc2, Wendy Barclay for a plasmid encoding the PB2 subunit of influenza B, Sylvie Van der Werf for a plasmid encoding the influenza C PB2 subunit and Caroline Demeret for plasmids encoding unrelevant Luc1/Luc2 tagged proteins. C.M. and B.D. acknowledge support of the ANR-17-CE18-0006-01 program. J.M. acknowledges fellowships of the ANR program and the Animal Health Division of INRAE.

Conflicts of Interest: The authors declare no conflict of interest. The funders had no role in the design of the study, in the collection, analyses, or interpretation of data; in the writing of the manuscript, or in the decision to publish the results".

\section{References}

1. Palese, P.; Shaw, M.L. Orthomyxoviridae: The Viruses and Their Replication. In Fields virology, 6th ed.; Philadelphia : Wolters Kluwer Health/Lippincott Williams \& Wilkins, 2013; Vol. 1, pp. 1151-1185.

2. Boivin, S.; Cusack, S.; Ruigrok, R.W.H.; Hart, D.J. Influenza A Virus Polymerase: Structural Insights into Replication and Host Adaptation Mechanisms. J. Biol. Chem. 2010, 285, 28411-28417, doi:10.1074/jbc.R110.117531.

3. Te Velthuis, A.J.W.; Fodor, E. Influenza Virus RNA Polymerase: Insights into the Mechanisms of Viral RNA Synthesis. Nat. Rev. Microbiol. 2016, 14, 479-493, doi:10.1038/nrmicro.2016.87.

4. Poon, L.L.; Pritlove, D.C.; Fodor, E.; Brownlee, G.G. Direct Evidence That the Poly(A) Tail of Influenza A Virus MRNA Is Synthesized by Reiterative Copying of a U Track in the Virion RNA Template. J Virol 1999, 73, 3473-3476, doi:10.1128/JVI.73.4.34733476.1999.

5. Krischuns, T.; Lukarska, M.; Naffakh, N.; Cusack, S. Influenza Virus RNA-Dependent RNA Polymerase and the Host Transcriptional Apparatus. Annu Rev Biochem 2021, 90, 321-348, doi:10.1146/annurev-biochem-072820-100645. 
6. Wandzik, J.M.; Kouba, T.; Karuppasamy, M.; Pflug, A.; Drncova, P.; Provaznik, J.; Azevedo, N.; Cusack, S. A Structure-Based Model for the Complete Transcription Cycle of Influenza Polymerase. Cell 2020, S0092867420303895, doi:10.1016/j.cell.2020.03.061.

7. Lamb, R.A.; Choppin, P.W. Synthesis of Influenza Virus Polypeptides in Cells Resistant to Alpha-Amanitin: Evidence for the Involvement of Cellular RNA Polymerase II in Virus Replication. Journal of Virology 1977, 23, 816-819, doi:10.1128/JVI.23.3.816819.1977.

8. Engelhardt, O.G.; Smith, M.; Fodor, E. Association of the Influenza A Virus RNA-Dependent RNA Polymerase with Cellular RNA Polymerase II. JVI 2005, 79, 5812-5818, doi:10.1128/JVI.79.9.5812-5818.2005.

9. Walker, A.P.; Fodor, E. Interplay between Influenza Virus and the Host RNA Polymerase II Transcriptional Machinery. Trends in Microbiology 2019, 27, 398-407, doi:10.1016/j.tim.2018.12.013.

10. Cramer, P.; Bushnell, D.A.; Kornberg, R.D. Structural Basis of Transcription: RNA Polymerase II at 2.8 Angstrom Resolution. Science 2001, 292, 1863-1876, doi:10.1126/science.1059493.

11. Cramer, P. Organization and Regulation of Gene Transcription. Nature 2019, 573, 45-54, doi:10.1038/s41586-019-1517-4.

12. Nojima, T.; Gomes, T.; Grosso, A.R.F.; Kimura, H.; Dye, M.J.; Dhir, S.; Carmo-Fonseca, M.; Proudfoot, N.J. Mammalian NETSeq Reveals Genome-Wide Nascent Transcription Coupled to RNA Processing. Cell 2015, 161, 526-540, doi:10.1016/j.cell.2015.03.027.

13. Martínez-Alonso, M.; Hengrung, N.; Fodor, E. RNA-Free and Ribonucleoprotein-Associated Influenza Virus Polymerases Directly Bind the Serine-5-Phosphorylated Carboxyl-Terminal Domain of Host RNA Polymerase II. J. Virol. 2016, 90, 6014-6021, doi:10.1128/JVI.00494-16.

14. Lukarska, M.; Fournier, G.; Pflug, A.; Resa-Infante, P.; Reich, S.; Naffakh, N.; Cusack, S. Structural Basis of an Essential Interaction between Influenza Polymerase and Pol II CTD. Nature 2017, 541, 117-121, doi:10.1038/nature20594.

15. Serna Martin, I.; Hengrung, N.; Renner, M.; Sharps, J.; Martínez-Alonso, M.; Masiulis, S.; Grimes, J.M.; Fodor, E. A Mechanism for the Activation of the Influenza Virus Transcriptase. Molecular Cell 2018, 70, 1101-1110.e4, doi:10.1016/j.molcel.2018.05.011.

16. Fodor, E.; Devenish, L.; Engelhardt, O.G.; Palese, P.; Brownlee, G.G.; García-Sastre, A. Rescue of Influenza A Virus from Recombinant DNA. J Virol 1999, 73, 9679-9682, doi:10.1128/JVI.73.11.9679-9682.1999.

17. Le Goffic, R.; Bouguyon, E.; Chevalier, C.; Vidic, J.; Da Costa, B.; Leymarie, O.; Bourdieu, C.; Decamps, L.; Dhorne-Pollet, S.; Delmas, B. Influenza A Virus Protein PB1-F2 Exacerbates IFN-Beta Expression of Human Respiratory Epithelial Cells. J Immunol 2010, 185, 4812-4823, doi:10.4049/jimmunol.0903952.

18. Cassonnet, P.; Rolloy, C.; Neveu, G.; Vidalain, P.-O.; Chantier, T.; Pellet, J.; Jones, L.; Muller, M.; Demeret, C.; Gaud, G.; et al. Benchmarking a Luciferase Complementation Assay for Detecting Protein Complexes. Nat Methods 2011, 8, 990-992, doi:10.1038/nmeth.1773.

19. Da Costa, B.; Sausset, A.; Munier, S.; Ghounaris, A.; Naffakh, N.; Le Goffic, R.; Delmas, B. Temperature-Sensitive Mutants in the Influenza A Virus RNA Polymerase: Alterations in the PA Linker Reduce Nuclear Targeting of the PB1-PA Dimer and Result in Viral Attenuation. J Virol 2015, 89, 6376-6390, doi:10.1128/JVI.00589-15.

20. Shyu, Y.J.; Liu, H.; Deng, X.; Hu, C.-D. Identification of New Fluorescent Protein Fragments for Bimolecular Fluorescence Complementation Analysis under Physiological Conditions. Biotechniques 2006, 40, 61-66, doi:10.2144/000112036.

21. Leymarie, O.; Jouvion, G.; Hervé, P.-L.; Chevalier, C.; Lorin, V.; Lecardonnel, J.; Da Costa, B.; Delmas, B.; Escriou, N.; Le Goffic, R. Kinetic Characterization of PB1-F2-Mediated Immunopathology during Highly Pathogenic Avian H5N1 Influenza Virus Infection. PLoS One 2013, 8, e57894, doi:10.1371/journal.pone.0057894.

22. Nilsson-Payant, B.E.; Sharps, J.; Hengrung, N.; Fodor, E. The Surface-Exposed PA51-72-Loop of the Influenza A Virus Polymerase Is Required for Viral Genome Replication. J Virol 2018, 92, e00687-18, doi:10.1128/JVI.00687-18.

23. Munier, S.; Rolland, T.; Diot, C.; Jacob, Y.; Naffakh, N. Exploration of Binary Virus-Host Interactions Using an Infectious Protein Complementation Assay. Mol Cell Proteomics 2013, 12, 2845-2855, doi:10.1074/mcp.M113.028688.

24. Cramer, P. RNA Polymerase II Structure: From Core to Functional Complexes. Curr Opin Genet Dev 2004, 14, 218-226, doi:10.1016/j.gde.2004.01.003.

25. Huet, S.; Avilov, S.V.; Ferbitz, L.; Daigle, N.; Cusack, S.; Ellenberg, J. Nuclear Import and Assembly of Influenza A Virus RNA Polymerase Studied in Live Cells by Fluorescence Cross-Correlation Spectroscopy. JVI 2010, 84, 1254-1264, doi:10.1128/JVI.01533-09.

26. Pflug, A.; Guilligay, D.; Reich, S.; Cusack, S. Structure of Influenza A Polymerase Bound to the Viral RNA Promoter. Nature 2014, 516, 355-360, doi:10.1038/nature14008.

27. Pflug, A.; Lukarska, M.; Resa-Infante, P.; Reich, S.; Cusack, S. Structural Insights into RNA Synthesis by the Influenza Virus Transcription-Replication Machine. Virus Research 2017, 234, 103-117, doi:10.1016/j.virusres.2017.01.013.

28. Sugiyama, K.; Obayashi, E.; Kawaguchi, A.; Suzuki, Y.; Tame, J.R.H.; Nagata, K.; Park, S.-Y. Structural Insight into the Essential PB1-PB2 Subunit Contact of the Influenza Virus RNA Polymerase. EMBO J 2009, 28, 1803-1811, doi:10.1038/emboj.2009.138.

29. Meka, H. Crystal Structure and RNA Binding of the Rpb4/Rpb7 Subunits of Human RNA Polymerase II. Nucleic Acids Research 2005, 33, 6435-6444, doi:10.1093/nar/gki945. 
30. Allepuz-Fuster, P.; Martínez-Fernández, V.; Garrido-Godino, A.I.; Alonso-Aguado, S.; Hanes, S.D.; Navarro, F.; Calvo, O. Rpb4/7 Facilitates RNA Polymerase II CTD Dephosphorylation. Nucleic Acids Research 2014, 42, 13674-13688, doi:10.1093/nar/gku1227.

31. Allepuz-Fuster, P.; O’Brien, M.J.; González-Polo, N.; Pereira, B.; Dhoondia, Z.; Ansari, A.; Calvo, O. RNA Polymerase II Plays an Active Role in the Formation of Gene Loops through the Rpb4 Subunit. Nucleic Acids Res 2019, 47, 8975-8987, doi:10.1093/nar/gkz597.

32. Kimura, M.; Suzuki, H.; Ishihama, A. Formation of a Carboxy-Terminal Domain Phosphatase (Fcp1)/TFIIF/RNA Polymerase II (Pol II) Complex in Schizosaccharomyces Pombe Involves Direct Interaction between Fcp1 and the Rpb4 Subunit of Pol II. Mol Cell Biol 2002, 22, 1577-1588, doi:10.1128/MCB.22.5.1577-1588.2002.

33. Mitsuzawa, H.; Kanda, E.; Ishihama, A. Rpb7 Subunit of RNA Polymerase II Interacts with an RNA-Binding Protein Involved in Processing of Transcripts. Nucleic Acids Res 2003, 31, 4696-4701, doi:10.1093/nar/gkg688.

34. Chen, Y.; Yamaguchi, Y.; Tsugeno, Y.; Yamamoto, J.; Yamada, T.; Nakamura, M.; Hisatake, K.; Handa, H. DSIF, the Paf1 Complex, and Tat-SF1 Have Nonredundant, Cooperative Roles in RNA Polymerase II Elongation. Genes Dev 2009, 23, 2765-2777, doi:10.1101/gad.1834709.

35. Armache, K.-J.; Mitterweger, S.; Meinhart, A.; Cramer, P. Structures of Complete RNA Polymerase II and Its Subcomplex, Rpb4/7. J. Biol. Chem. 2005, 280, 7131-7134, doi:10.1074/jbc.M413038200.

36. Calvo, O. RNA Polymerase II Phosphorylation and Gene Looping: New Roles for the Rpb4/7 Heterodimer in Regulating Gene Expression. Curr Genet 2020, 66, 927-937, doi:10.1007/s00294-020-01084-w.

37. Choder, M. Rpb4 and Rpb7: Subunits of RNA Polymerase II and Beyond. Trends Biochem Sci 2004, 29, 674-681, doi:10.1016/j.tibs.2004.10.007.

38. Richard, S.; Gross, L.; Fischer, J.; Bendalak, K.; Ziv, T.; Urim, S.; Choder, M. Numerous Post-Translational Modifications of RNA Polymerase II Subunit Rpb4/7 Link Transcription to Post-Transcriptional Mechanisms. Cell Rep 2021, 34, 108578, doi:10.1016/j.celrep.2020.108578.

39. Vos, S.M.; Pöllmann, D.; Caizzi, L.; Hofmann, K.B.; Rombaut, P.; Zimniak, T.; Herzog, F.; Cramer, P. Architecture and RNA Binding of the Human Negative Elongation Factor. Elife 2016, 5, e14981, doi:10.7554/eLife.14981.

40. Yamaguchi, Y.; Takagi, T.; Wada, T.; Yano, K.; Furuya, A.; Sugimoto, S.; Hasegawa, J.; Handa, H. NELF, a Multisubunit Complex Containing RD, Cooperates with DSIF to Repress RNA Polymerase II Elongation. Cell 1999, 97, 41-51, doi:10.1016/s00928674(00)80713-8. 